\title{
Observations on vitamin $B_{12}$ in serum and cerebrospinal fluid in multiple sclerosis
}

\author{
W. BASIL, J. K. BROWN, AND D. M. MATTHEWS \\ From the Department of Chemical Pathology, Institute of Neurology, the National Hospital, \\ Queen Square, London
}

SYNOPSIS There are several claims that $B_{12}$ concentrations in serum and cerebrospinal fluid are grossly abnormal in multiple sclerosis, but results are conflicting. This paper reports measurements of these concentrations in 40 serum samples and 23 samples of lumbar cerebrospinal fluid from cases of multiple sclerosis, and in appropriate controls, using Euglena gracilis, z strain. The serum concentrations were found to be normal; the mean concentration in cerebrospinal fluid was slightly reduced, but all values were within the control range.

In both control samples and samples from cases of multiple sclerosis, the $\mathrm{B}_{12}$ concentration in lumbar cerebrospinal fluid was correlated with the concentration in serum. There was no correlation between $B_{12}$ concentration and total protein in cerebrospinal fluid.

A number of estimations of serum $B_{12}$ were also made with Lactobacillus leichmannii, after extraction in the presence and absence of cyanide. These showed a difference between cases of multiple sclerosis and controls, one interpretation of which might be that the serum in multiple sclerosis contains an abnormally low concentration of hydroxocobalamin.

Several investigations have been made of the vitamin $\mathrm{B}_{12}$ concentration in body fluids in multiple sclerosis, but the results are completely conflicting. Sobotka, Christoff, and Baker (1958) and Sobotka, Baker, Frank, Maker, and Margulies (1960), using Ochromonas malhamensis, found large increases in serum and cerebrospinal fluid $B_{12}$ in about a third of their cases. On the other hand O'Connor, Davis, Langworthy, and Chow (1960), using Lactobacillus leichmannii, reported that the serum $\mathrm{B}_{12}$ was low in multiple sclerosis, half of their patients having concentrations within the pernicious anaemia range, and Bauer and Heinrich (1961), using Euglena gracilis, also reported very low serum values. Gjertsen and Schrumpf (1962), using Euglena gracilis, found low values in cerebrospinal fluid. Grann and Glass (1961), using Ochromonas malhamensis, found normal serum $B_{12}$ concentrations in the majority of cases; three out of 21 cases had elevated concentrations, but two of these had been given parenteral $B_{12}$ : one case had a subnormal concentration. The majority of their patients had normal $\mathrm{B}_{12}$ absorption as estimated by hepatic uptake. Worm-Petersen (1962), using Euglena gracilis, $\mathrm{z}$ strain, found no significant difference in cerebrospinal fluid and serum $B_{12}$ concentrations Received for publication 2 June 1964. between cases of multiple sclerosis and controls. Kidd, Gould, and Thomas (1963), using Euglena gracilis, $\mathrm{z}$ strain, estimated $\mathrm{B}_{12}$ in serum and cerebrospinal fluid in a variety of neurological disorders, including three cases of multiple sclerosis; in two of these the values were normal, though in one there was a slight rise in $\mathrm{B}_{12}$ concentration in cerebrospinal fluid. The work reported here was undertaken in the hope of clarifying this confused position, and in order to gain information about the relationship of the $B_{12}$ concentration in cerebrospinal fluid to that in serum and to cerebrospinal fluid protein. The majority of the estimations were carried out with Euglena gracilis, $\mathrm{z}$ strain. In some cases, serum estimations were also made with Lactobacillus leichmannii, after extraction in the presence and absence of cyanide. This was done in view of the possibility that the difference between these two values indicates the relative proportions of cyanocobalamin and hydroxocobalamin in the serum and the suggestion that a disturbance of cyanide metabolism, involving an alteration in these proportions, might be concerned in the pathogenesis of demyelination (Smith, 1961). Since the present work was completed, Simpson (1964), using Euglena gracilis, $\mathrm{z}$ strain, has reported no significant dif- 
ference between cerebrospinal fluid and serum $\mathbf{B}_{\mathbf{1 2}}$ concentrations in multiple sclerosis and control values.

\section{MATERIALS AND METHODS}

Estimations of levels of vitamin $B_{12}$ in serum and cerebrospinal fluid were made by a modification of the method of Hutner, Bach, and Ross (1956) with Euglena gracilis, $\mathrm{z}$ strain. The cerebrospinal fluid, which was not centrifuged, was usually assayed at a dilution of 1 in 6 , though when the amount available was small, dilutions of 1 in 8 or 1 in 12 were used. Serum estimations with Lactobacillus leichmannii were made as described by Matthews (1962); each sample was divided into two parts, one of which was assayed after extraction by heating for $\mathbf{3 0}$ minutes with acetate buffer $p \mathrm{H} 4.6$ containing $20 \mathrm{mg}$. $\mathrm{NaCN}$ per litre, and the other after extraction in the same way with buffer containing no cyanide.

The control group for serum $B_{12}$ was made up of hospital in-patients and out-patients aged 18 to 75 , none of whom were known to have any condition affecting the serum $B_{12}$ concentration. The group of cases of multiple sclerosis was made up of patients aged 17 to 57 , in all of whom the diagnosis was firmly established. If the patients had received $B_{12}$, this was noted. Samples of lumbar cerebrospinal fluid were divided into three groups.

1 THE CONTROL GROUP In this, the cerebrospinal fluid protein concentration was not more than $50 \mathrm{mg}$. per $100 \mathrm{ml}$. and the cell count, Pandy Nonne-Apelt and Lange tests were normal, though many of the patients had organic neurological lesions.

2 A 'PATHOLOGICAL' GROUP In this, the cerebrospinal fluid protein concentration ranged from 55 to $500 \mathrm{mg}$. per $100 \mathrm{ml}$. and the white cell count up to 500 per c. mm., and in many cases there were other abnormal findings; the patients suffered from a variety of neurological disorders.

\section{SAMPLES FROM CASES OF MULTIPLE SCLEROSIS}

In addition, a small number of samples of ventricular cerebrospinal fluid were examined. These came from patients with a variety of neurological disorders; the protein concentration ranged up to $20 \mathrm{mg}$. per $100 \mathrm{ml}$.,

and in some cases there were other abnormal findings. groups 1 and 2 or ventricular cerebrospinal fluid were $\overrightarrow{\vec{F}}$ known to have any condition affecting the serum $B_{12} \stackrel{\oplus}{\oplus}$ concentration.

STATISTICAL METHODS The distribution of $\mathbf{B}_{12}$ concen- $\overline{\bar{N}}$ trations in serum and cerebrospinal fluid fits a logarithmic $\widehat{\nabla}$ distribution better than a normal one, as Worm-Petersen 2 (1962) points out, and Simpson (1964) has used the logarithms of the observations for all calculations. We have $\overrightarrow{ }$ also done this in addition to making calculations on the usual arithmetical basis. In the majority of instances the $\vec{\omega}$ logarithmic method made no essential difference to the $\stackrel{\circ}{\circ}$ results of significance tests, and in such cases the logarith-용 mic results are omitted for the sake of clarity. When there was an appreciable difference, logarithmic results $\infty$ are given. The significance of differences between means $\omega$ was assessed by the $t$ test.

\section{RESULTS}

The $B_{12}$ concentrations obtained in serum and lumber cerebrospinal fluid are shown in Table $I$. The mean concentration in 'pathological' cerebro- $\vec{\theta}$ spinal fluid was significantly higher than that in the ज़ control group $((\mathrm{P}<0.05>0.02)$. In patients with multiple sclerosis, none of whom were known to have had $B_{12}$, the mean serum $B_{12}$ concentration was not significantly different from that in controls, but the mean concentration in cerebrospinal fluid was $\Phi$ significantly lower (controls, mean of logs $1.33, \overrightarrow{\vec{T}}$ S.E. 0.03 ; multiple sclerosis, mean of logs $1 \cdot 18$, S.E. $0.05 ; P<0.02>0.01)$ and all the values except one were in the lower part of the control range (Fig. 1). In both control subjects and the cases of multiple sclerosis, there was a correlation between the $\mathrm{B}_{12}$ ? concentration in serum and cerebrospinal fluid (Figs. 2 and 3). In controls the ratio of $\mathrm{B}_{12}$ concentration in cerebrospinal fluid to serum concentration in 0 paired samples was $5.6 \%$, whereas in multiple sclerosis it was only $3.8 \%$. Six other cases of multiple 음 sclerosis were known to have been given parenteral $\rightarrow$ $B_{12}$ within three months of the estimations. In these,

TABLE I

$B_{12}$ CONCENTRATIONS IN SERUM AND LUMBAR CEREBROSPINAL FLUID USING EUGLENA GRACILIS, Z STRAIN

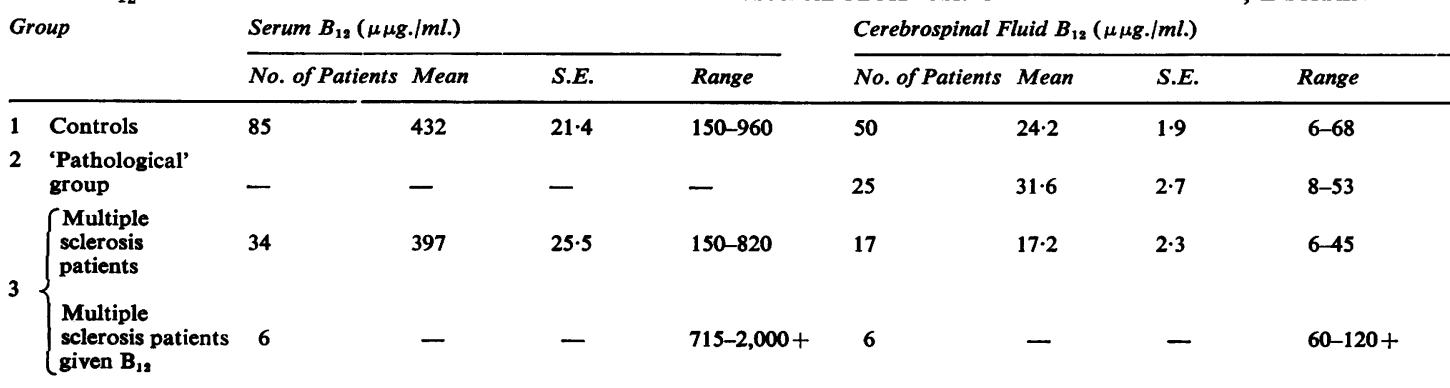




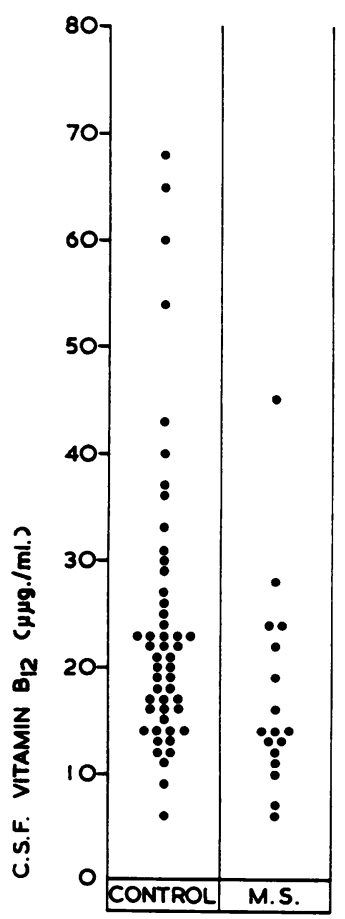

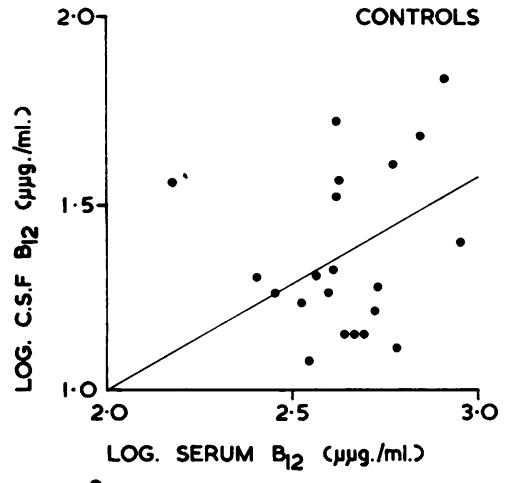

FIG. 2 .

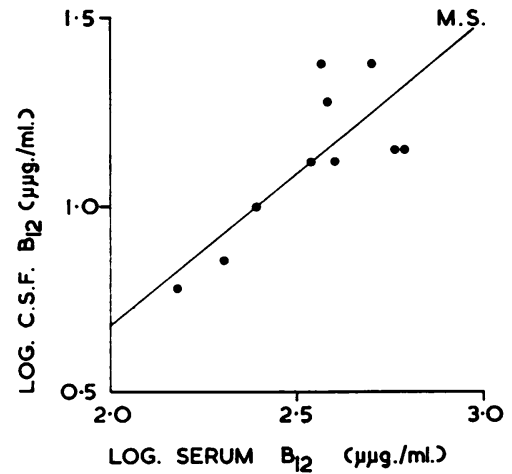

FIG. 3 .

FIG. 1. Vitamin $B_{12}$ concentrations in lumbar cerebrospinal fluid in control samples and in multiple sclerosis.

FIG. 2. Correlation between $\log$ cerebrospinal fluid $B_{12}$ and $\log$ serum $B_{12}$ in controls. The calculated regression line is shown $\left(r^{12}=0.46 ; P<0.05>0.02\right)$.

FIG. 3. Correlation between $\log$ cerebrospinal fluid $B_{12}$ and $\log$ serum $B_{12}$ in multiple sclerosis. The calculated regression line is shown $(r=0.77 ; P<0.01>$ 0.001 ).

FIG. 1.

the serum and cerebrospinal fluid concentrations were high, five of the serum values and five of the cerebrospinal fluid values being above the upper limit of the control range. No correlation was found between the $B_{12}$ concentration in cerebrospinal fluid, and total protein or cell count, in any group. In ventricular cerebrospinal fluid, the mean $B_{12}$ concentration $(8.4 \mu \mu \mathrm{g}$. per $\mathrm{ml}$., S.E. $1 \cdot 7, \mathrm{n}=10)$ was significantly lower than in lumbar control samples $(\mathrm{P}<0.001)$.

SERUM $B_{12}$ CONCENTRATIONS OBTAINED WITH Lactobacillus leichmannii AFTER EXTRACTION IN PRESENCE AND ABSENCE OF CYANIDE The serum $B_{12}$ concentration obtained by this method in the presence of cyanide measures the total serum $B_{12}$. In 75 control patients the mean value was $463 \mu \mu \mathrm{g}$./ml. (range 115950 , S.E. 22). In 13 patients with multiple sclerosis, none of whom were known to have had $B_{12}$, the mean value was $481 \mu \mu \mathrm{g} . / \mathrm{ml}$. (range 220-700, S.E. 36 ); the difference is not significant. Values obtained in the absence of cyanide are lower than those in the presence of cyanide, for reasons discussed below. In 38 control sera, the mean value obtained wthout cyanide was $53.5 \%$ (range $28-75 \%$, S.E. 2.0 ) of that obtained with cyanide. In the cases of multiple sclerosis (with a similar age and sex distribution), the mean value obtained without cyanide was $64.1 \%$ (range $51-75 \%$ ) of that obtained with cyanide (Fig. 4). The difference is significant $(\mathrm{P}<$ $0.01>0.001)$.

\section{DISCUSSION}

The published values for $B_{12}$ concentration in lumbar cerebrospinal fluid vary very widely $(e . g$. Ross, 1950 ; Sobotka, Baker, and Frank, 1960; Bauer and Heinrich, 1961; Yavorkovsky and Mai, 1961; Gjersten and Schrumpf, 1962) but the control values we have found agree well with those of Herbert and Zalusky (1961), Worm-Petersen (1962), and Kidd, et al. (1963). The finding of a correlation between $B_{12}$ concentrations in serum and cerebrospinal fluid confirms the reports of Worm-Petersen (1962) and of Simpson (1964). We also agree with these authors on the lack of correlation between $B_{12}$ concentration and total protein in cerebrospinal fluid, though this conflicts with the report of Yavorkovsky and Mai (1961). Values for $B_{12}$ in ventricular cerebrospinal fluid have not been reported previously as far as we know. It is not obvious why the $B_{12}$ concentration is lower in ventricular than in lumbar fluid. The slight increase in $\mathbf{B}_{12}$ concentration in pathological lumbar cerebrospinal fluid (excluding samples from multiple 


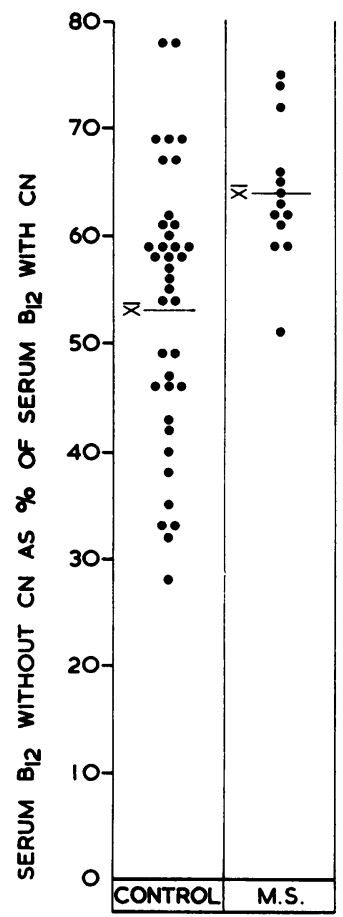

FIG. 4. Control sera and sera from multiple sclerosis: the $B_{12}$ value obtained after extraction without cyanide as a percentage of that obtained after extraction with cyanide (Lactobacillus leichmannii).

sclerosis) may reflect an increase in the permeability of the blood-brain barrier.

The present work has produced no evidence of major alterations in $B_{12}$ in serum and cerebrospinal fluid in multiple sclerosis, but it does suggest that there are minor abnormalities. High values were only found when the patients were known to have received $B_{12}$. Excluding these cases, the total serum $B_{12}$ was found to be normal using two different assay organisms, and the cerebrospinal fluid concentrations were all within the control range, though the mean was significantly lower than in controls. The tendency for levels of $B_{12}$ in cerebrospinal fluid to be reduced in multiple sclerosis contrasts with the tendency for it to be increased in the group of miscellaneous neurological disorders. The results suggest that at a given concentration of serum $B_{12}$ the concentration of $B_{12}$ in cerebrospinal fluid is slightly lower in multiple sclerosis than in controls. No explanation for this can be suggested without further investigation, and it is unlikely to be explained until the factors governing the concentration of $B_{12}$ in cerebrospinal fluid are better understood. These probably include not only the concentration of $\mathrm{B}_{12}$ in serum, but the permeability of the bloodbrain barrier, the $\mathbf{B}_{12}$-binding capacity of the cerebrospinal fluid (Meyer, Bertcher, and Mulzac, 1959; Clausen and Munkner, 1962; Worm-Petersen, 1962) and perhaps utilization by the brain. Our finding of

normal concentrations of total serum $B_{12}$ in multiple sclerosis confirms the reports of Grann and Glass: (1961) and Worm-Petersen (1962). Simpson(1964)alsas found that the mean serum concentration was noto significantly different from that in controls, but int his series there were several values below the lowerw limit of the control range. There is no major disagreement between the present finding of a moderate reduction in cerebrospinal fluid $\mathrm{B}_{12}$ concentration and the results of Worm-Petersen and of Simpson, for both these authors found that the mean cerebro $-\vec{A}$ spinal fluid $B_{12}$ concentration in multiple sclerosis was slightly lower than in controls, though thes difference was not statistically significant. We cannot confirm the reports of very low serum and cerebrospinal fluid $B_{12}$ concentrations. The reports of high concentrations may well be due to the administration of $\mathrm{B}_{12}$, as the investigators concerned do not stateo whether or not this had been given.

The results of the measurements of serum $B_{12}$ with Lactobacillus leichmannii after extraction withou cyanide show a small but significant difference between cases of multiple sclerosis and controls. ItS has been shown that extraction of serum in the absence of cyanide causes a large though variables loss of $B_{12}$ in association with the protein precipi tate, which may be in the form of hydroxocobalamine (Matthews $1961 ; 1962$ ), and it is possible that the serum $B_{12}$ value obtained in the absence of cyanideo represents serum cyanocobalamin while the addi $\overrightarrow{\overrightarrow{0}}$ tional $B_{12}$ obtained in the presence of cyanide repre 3 sents serum hydroxocobalamin. This hypothesis which has recently received some further experimental support (Anderson, 1964), is energetically advocated by Smith (1961) who claims that patients with tobacco amblyopia have an abnormally low 3 proportion of hydroxocobalamin in the blood. Interpreted in this way, the present results wouls indicate that in multiple sclerosis the blood tends to contain less hydroxocobalamin and more cyanocobalamin than normal. Unfortunately, it is not ye possible to interpret the results with certainty, as the forms in which $\mathrm{B}_{12}$ exists in the blood are unknown $\mathrm{N}$ If the bulk of it is in the coenzyme form, the value obtained in the absence of cyanide may reflect not the concentration of cyanocobalamin in the bloof but the concentration of cyanide or thiocyanate? However, the finding of the difference that has beent described between patients with multiple sclerosis and control subjects indicates the desirability of further investigation.

Though $\mathrm{B}_{12}$ is often given in multiple sclerosis, there is little to suggest that it influences the course of the disease (Simpson, 1964), and the present worke has produced no evidence of overt $B_{12}$ deficiency in the condition. If the vitamin is to be given, there if 
a case for using hydroxocobalamin, which is retained in the body longer than cyanocobalamin. The present results might be regarded as additional justification for preferring hydroxocobalamin to the cyano-form.

We wish to thank members of the staff of the National Hospital for allowing us to study patients under their care, and Dr. P. K. Thomas for providing some of the specimens. We are also grateful to Mrs. V. Newbery for technical assistance, and to Miss June Quick for preparation of the figures.

\section{ADDENDUM}

Since this paper was submitted for publication, it has been shown (Lindstrand, 1964) that the bulk of the $\mathrm{B}_{12}$ in normal blood is in the form of methylcobalamin, and this finding must be taken into consideration in interpreting the present results. Further work on the inter-relationships between cyanide and $B_{12}$ in controls and patients with multiple sclerosis is to be published elsewhere (Matthews, Wilson, and Zilkha, 1965).

\section{REFERENCES}

Anderson, B. B. (1964). J. clin. Path., 17, 14.

Bauer, H., and Heinrich, H. C. (1961). In Chemical Pathology of the Nervous System, edited by J. Folch-Pi, p. 120. Pergamon Press, Oxford.

Clausen, J., and Munkner, T. (1962). Acta path. microbiol. scand., $54,129$.

Gjertsen, F. A., and Schrumpf (1962). Nord. Med., 67, 162.

Grann, V., and Glass, G. B. J. (1961). J. Lab. clin. Med., 57, 562.

Herbert, V., and Zalusky, R. (1961). Fed. Proc., $20,453$.

Hutner, S. H., Bach, M. K., and Ross, G. I. M. (1956). J. Protozool., 3, 101.

Kidd, H. M., Gould, C. E. G., and Thomas, J. W. (1963). Canad. med. Ass. J., 88, 876.

Lindstrand, K. (1964). Nature (Lond.), 204, 188.

Matthews, D. M. (1961). Lancet, 1, 1289.

- (1962). Clin. Sci., 22, 101.

—, Wilson, J., and Zilkha, K. J. (1965). J. Neurol. Neurosurg. Psychiat. In press.

Meyer, L. M., Bertcher, R. W., and Mulzac, C. (1959). Proc. Soc. exp. Biol. (N.Y.), 100, 607.

O'Connor, J. S., Davis, R. L., Langworthy, O. R., and Chow, B. F. (1960). Ibid., 103, 180.

Ross, G. I. M. (1950). Nature (Lond.), 166, 270.

Simpson, C. A. (1964). J. Neurol. Neurosurg. Psychiat., 27, 174.

Smith, A. D. M. (1961). Lancet, 1, 1001.

Sobotka, H., Baker, H., and Frank, O. (1960). Proc. Soc. exp. Biol. $(N . Y), 103,801$.

—, Christoff, N., and Baker, H. (1958). Ibid., 98. 534.

-, Baker, H., Frank, O., Maker, H., and Margulies, M. E. (1960). J. Mt Sinai Hosp., $27,618$.

Worm-Petersen, J. (1962). Acta neurol. scand., 38, 241.

Yavorkovsky, L. I., and Mai, L. A. (1961). Vop. med. Khim., 7, 25. Quoted by Kidd et al., (1963). 